\title{
Simulation of Lunar Surface Communications Network Exploration Scenarios
}

\author{
Thomas W. Linsky* and Kul B. Bhasin ${ }^{\dagger}$ \\ NASA Glenn Research Center, Cleveland, OH, 44135, United States \\ Alex White ¿ $^{\ddagger}$ \\ Highland Systems, Inc., Arlington, VA, 22201, United States \\ Srihari Palangala ${ }^{\S}$ \\ OPNET Technologies, Inc., Bethesda, MD, 20814, United States
}

\begin{abstract}
NASA is initiating a new program to explore the Moon, Mars, and beyond with safe, sustainable, and affordable missions. These new exploration missions will require a more reliable and flexible communications network than used in today's science missions to serve as the medium for voice, video, and data exchange on the surface. This integrated network will enable safety, collaboration, and autonomy to address the emerging requirements of remote human exploration. It will span communications links with vastly differing time delays and intermittent connectivity. For example, local communications links among nearby entities on the surface of the Moon will include more unpredictable connectivity, less time delay, and more relative node mobility than long range communications between planets and moons. These heterogeneous links will coexist in one complex networked system of systems architecture.

Simulation and modeling of surface-based communications networks provides a rapid and cost effective means of requirement analysis, protocol assessments, and tradeoff studies. Robust testing is especially important for exploration systems, where the cost of deployment is high and systems cannot be easily replaced or repaired. However, simulation of the envisioned exploration networks cannot be achieved using commercial off the shelf network simulation software. The surface network environment, which includes a high degree surface mobility with changing line-of-sight and unpredictable connectivity, and the complexity of the nodes involved differentiates surface simulation from terrestrial simulation. In addition, models for the nonstandard, non-COTS protocols used aboard space systems are not readily available.

This paper will address the simulation of realistic scenarios representative of the activities which will take place on the surface of the Moon, including selection of candidate network architectures, and the development of an integrated simulation tool using OPNET modeler capable of faithfully modeling those communications scenarios in the variable delay, dynamic surface environments. Scenarios for exploration missions, OPNET development, limitations, and simulation results will be provided and discussed.
\end{abstract}

\section{Introduction}

$\mathrm{T}$ HE Vision for Space Exploration ${ }^{1}$ refocused NASA to a new initiative to explore the solar system with safe, sustainable, and affordable missions. To achieve such missions and address the problems encountered during human exploration of unknown areas, new systems and technologies are required which can

\footnotetext{
*Telecommunications Engineer, Communications ComputingElectronics and Imaging Projects, 21000 Brookpark Rd., Cleveland, $\mathrm{OH}, 44135$

${ }^{\dagger}$ Manager, Communications Computing Electronics and Imaging Projects, 21000 Brookpark Rd., Cleveland, OH, 44135 AIAA Member

${ }^{\ddagger}$ Engineer, 3240 Wilson Blvd \#210, Arlington, VA, 22201

$\S$ Engineer, 7255 Woodmont Avenue, Bethesda, MD, 20814
} 
systemmatically extend the human presence in a safe and cost-effective manner. Exploration missions of the future will require a more reliable and efficient communications network than used in today's science missions to serve as the medium for voice, video, and data exchange. The network will integrate communications, navigation, power, and computing technologies to address the emerging requirements of remote human exploration, enabling safety, collaboration, and autonomy. It will span communications links with vastly differing time delays, noise levels, interference levels, and intermittent connectivity. For example, communications between large assets on the surface of the Moon will include more power, longer distances, higher data rates, and less relative node mobility than communications between small, mobile nodes such as robots. These heterogeneous links will reside together in one surface network, and that surface network will interface seamlessly with the other networks defined in the exploration system of systems. ${ }^{2}$

NASA's exploration roadmap calls for manned missions to the Lunar surface by $2020 .^{3}$ The initial missions will be similar to the Apollo missions, with no pre-deployed assets and the capability for landing anywhere on the Moon's surface for a duration of four to seven days. ${ }^{2}$ As the exploration capability matures, the Lunar missions will be expanded for use as a Mars testbed. An infrastructure consisting of power, in situ resource utilization, and communications will be pre-deployed on the surface, and the missions will increase in duration to between 42 and 98 days. ${ }^{2}$ The surface network will play an integral role in both the early and later Lunar missions and Mars missions, enabling collaboration between astronauts and advanced monitoring and control capabilities.

A realistic surface mission might include four astronauts, four robotic assistants, one rover, one habitat, and one lander in a 90 day mission. ${ }^{2}$ The communications architecture will build upon the success of the Mars Exploration Rovers by utilizing satellite relays ${ }^{3}$ rather than direct links to Earth to achieve higher data rates and lower power consumption. ${ }^{4}$ In order to reduce costs, leverage a large talent base of engineers, and provide maximum reliability, NASA will assess modified commercial off-the-shelf (COTS) protocols and hardware to leverage in the communications system with the goals of modularity, affordability, and interoperability. ${ }^{4}$ Near-term future missions will use similar technology to that used in today's missions: S-Band links and bent pipe relays, but no advanced network layer protocols. However, Mars missions, long duration Lunar missions missions, and a sustained human presence on the Moon may attempt to heavily leverage network protocols. ${ }^{5}$

Simulation and modeling of these new and untested communications networks provides a fast, inexpensive means of assessing mission requirements and performing tradeoff studies and performance assessments on critical protocols and network architectures. Robust testing is especially important for space exploration systems, where the cost of deployment is high and systems cannot be easily replaced or reconfigured. Simulation can be incorporated into all stages of the technology development process. However, simulation of the envisioned exploration networks poses challenges which cannot be addressed using commercial off the shelf (COTS) network simulation software. The surface network environment, which includes a high degree surface mobility with changing line-of-sight and unpredictable connectivity, and the complexity of the nodes involved make surface simulation unique and different from terrestrial simulation. In addition, the nonstandard, non-COTS protocols used aboard space systems are not modeled in COTS network simulators.

Even with simulation solutions for the problems above, testing of the surface networks remains a problem. Because this simulator will be used to test protocols and architectures in the context of a specific environment (the Lunar surface), accurate and comprehensive testing scenarios are important to maximize the reliablilty of the system when it is deployed. Countless possible testing scenarios exist and it is difficult to predict the Lunar infrastructure fifteen years into the future. As a result, an effective simulation tool must be capable of evolving to support new technologies and capabilities.

\section{Assumed Surface Communications Requirements}

The surface network will consist of a number of nodes (i.e. anything requiring communications) interconnected into a network. Nodes might include the habitat, lander, astronauts, rovers, robots, science instruments, controls, sensors, and cameras. Nodes will use the surface network to communicate with one another. A network architecture provides a high degree of flexibility and scalability to the surface operations and, because it can function effectively without a direct link to Earth and minimal human intervention to support autonomous and semi-autonomous surface operations.

The surface network, shown in figure 1 will consist of a number of nodes (i.e., anything requiring communications) interconnected into a network. These nodes might include the habitat, lander, astronauts, rovers, 


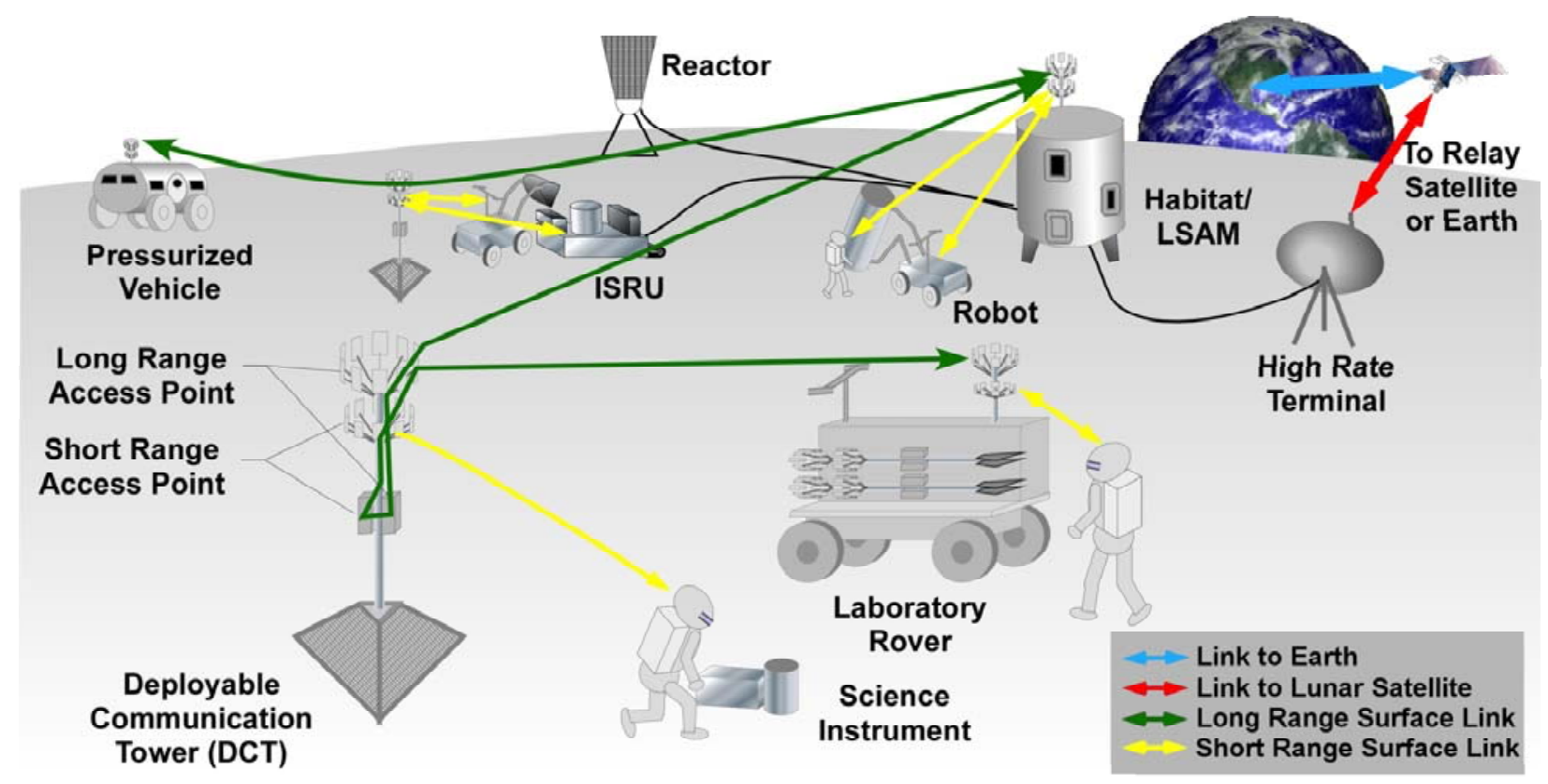

Figure 1. Lunar exploration operational communications scenario.

robots, science instruments, controls, sensors, and cameras. Nodes will use the surface network to communicate with one another, and each node needs a diverse application set to achieve its function. The surface network must integrate multiple applications with greatly differing requirements and seamlessly interface with the terrestrial and in-space networks planned by NASA. ${ }^{6}$ Some applications require more bandwidth and have different communications durations than others. Some require real-time interaction while others do not. Errors and packet loss are tolerable in some streams, but not in others. Streaming video, for example, can handle moderate data loss but requires high data rates and minimal variation in delay (i.e., jitter), while text e-mails require reliable data delivery with no packet loss but places no constraints on delay or jitter.

Because exploration capability will be built out gradually into a system of systems, the surface network must seamlessly interface with the system of systems elements, which may be built in different time frames from the surface systems. ${ }^{6}$ The surface network will interface with: the crew transportation system, which will transport the crew from Earth to their destination; the ground support system, which will provide the ability to manage flight systems, as well as land, recover, refurbish, or dispose of those systems; the inspace support system, which will provide mission support from space, including satellite relays; the robotic precursor system, which will use robotic technology to provide measurements, technology demonstrations, and infrastructure in preparation for human missions; and the cargo delivery system, which will transport un-crewed mission elements to their destination. ${ }^{2}$

The crew will likely spend a substantial amount of time operating and maintaining systems during the first Lunar missions. As the exploration capability shifts toward long duration Lunar missions and Mars missions, that time is expected to decrease as autonomous and semi-autonomous systems are introduced. ${ }^{7}$ Distributed, autonomous communications will be required for Mars missions, when large time delays prohibit real-time interactions with Earth. The surface network will provide the ability for autonomous node discovery and configuration, as well as autonomous data forwarding.

The enormous cost of transporting objects into Earth orbit and beyond will play a crucial role in the design of exploration missions. To mitigate the high per-pound mission cost, all elements involved in the exploration will be minimized with respect to mass and volume. Because of the stringent mass and volume constraints, most surface network nodes will not be capable of high-throughput power generation and high power communications. Network nodes will therefore require power-efficient components and power-aware protocols to enable a high performance, power-efficient exploration system. Alternative methods of reducing mass and volume, such as the reduction of cabling through wireless communications and power transfer, will increase the dependency of the system on wireless technologies. ${ }^{8}$ 


\section{Simulation Tools}

Numerous simulation tools exist which can provide partial simulation of the applications, networking, and physical layer which will operate within the surface network. OPNET Modeler, ns-2, and Qualnet are common terrestrial network simulators, which lack some of the protocol support required for surface exploration missions. Satellite Tool Kit, HerTZ mapper, ICS telecom, and wWLAN toolbox provide tools for physical layer properties which can be used for links within the exploration network, but do not include network simulation support. While models for many of the applications for surface networks, such as e-mail and voice traffic, are readily available, application models for many applications, such as CFDP and robotic control, are not commercially available. No off the shelf simulation tool is capable of complete Lunar surface network simulation.

\section{Scenarios}

Because the surface network, shown in figure 1 is being designed specifically to support exploration, it is important to accurately characterize the exploration scenarios under which the network will operate. A networking protocol might perform exceptionally in simulations only to fail when deployed because it was not tested under the proper conditions. Simulation can play an important role in the communications network architecture development process, by providing detailed feedback on proposed infrastructures and systems early in the design process.

However, simulating these communications network architectures also poses significant challenges. Because the Lunar exploration missions remain in the pre-planning phases, it is not clear what communications nodes and activities they will entail. This can lead to considerable difficulty in development of accurate and relevant exploration scenarios for simulation. The simulation framework must possess the flexibility to quickly adapt to top level scenario changes with a minimum level of development effort.

\section{A. Scenario Development Methodology}

NASA's Exploration Systems Mission Directorate has begun to release top level requirements for the early (spiral 1) crew transportation system ${ }^{2}$ and robotic precursor systems. ${ }^{9}$ For systems for which no official requirements have been released, such as the destination surface system, the simulation scenarios were synthesized from multiple reliable sources of information. NASA has released a number of design reference misssions for Lunar and Martian exploration, which are used as baselines by technology developers and mission planners. They represent missions which might be similar to those which will be undertaken in the future. Because spiral 3 long duration Lunar missions will be used as a testbed for Mars exploration, it is assumed here that most of the surface scenarios in those missions

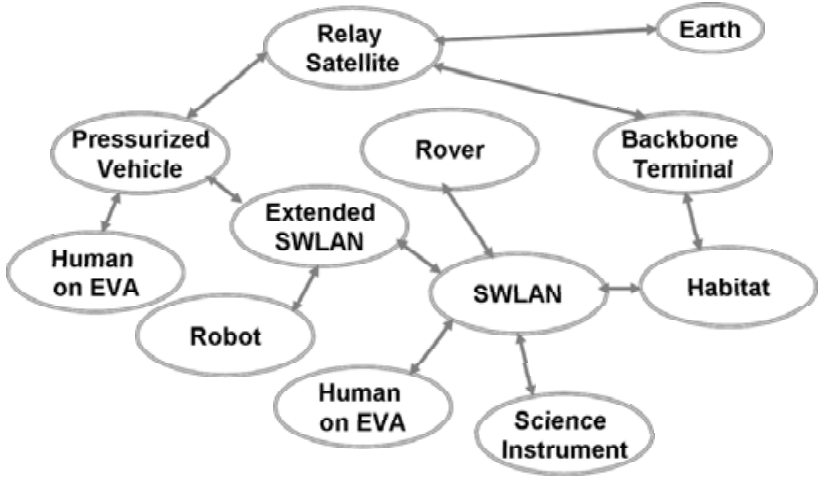

Figure 2. Operational nodes for Lunar exploration missions.

will be very similar to Mars missions. The Mars reference missions were therefore used in the development of long duration Lunar scenarios. Many of the design reference missions contain numerous commonalities, ranging from surface nodes, to in-mission activities, to mission concepts. The nodes and activities used in the simulation scenario were based on the design reference missions and on other key documents describing potential surface scenarios.

Once the nodes and activities were determined, more detailed information was obtained on the applications and communications required for the full functionality of each node. Nodes were broken down into component subsystems and each component was analyzed to assess the properties of the required communications. A robot, for example, has numerous components, including a camera, which will require communication of streaming video. Design reference missions, mission architecture concept sources were used to determine the components for each node, and technical documentation (e.g. terrestrial camera specs, ISS voice communications, etc) were used to assess the communications requirements for each component. 


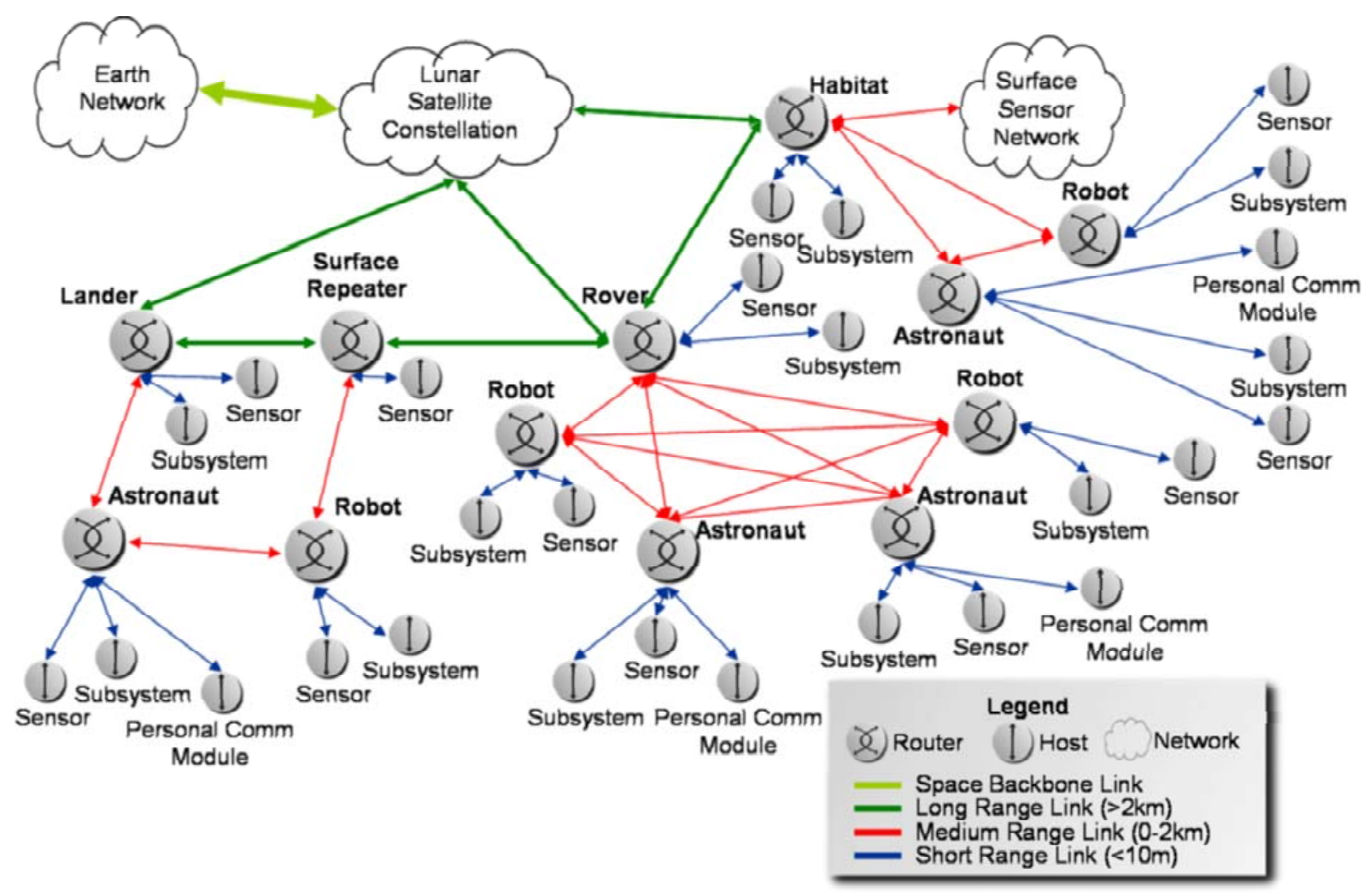

Figure 3. Lunar surface exploration scenario.

\section{B. Communications Nodes}

Exploration activities on the Lunar surface will involve a number of operational elements (nodes) which will provide the functionality required for safe and productive exploration. Figure 2 shows the operational nodes and for Lunar surface communications. Each node and some subsystems within each node will require communications with external nodes to achieve intelligent autonomous network operation. These nodes include: the Lunar surface access module, habitat, science instruments, rovers, robots, astronauts, and pressurized vehicles.

The Lunar Surface Access Module (LSAM) will provide crew habitation functions on the surface for four to six crew members during short stay (3-7 day) Lunar missions. ${ }^{2}$ It will also support EVA so that crew members can explore and, in long duration missions, transition to the habitat or other pre-deployed assets on the surface. ${ }^{2}$ The LSAM will include the communications hardware to establish multiple simultaneous bidirectional communications links to Earth, orbiting relays, and assets on the surface at both short $(<5 \mathrm{~km})$ and long $(<60 \mathrm{~km})$ distances. The LSAM design includes the following subsystems: avionics, environmental, airlock, vehicle command and control, communications, life support, thermal control, power, propulsion, crew accommodations, radiation protection, structures, and mechanisms. ${ }^{7,10,11}$ Each of these subsystems uses the surface network for interconnection and coordination with other systems and subsystems.

The Lunar Habitat is a pre-deployed surface habitation and science laboratory unit which provides habitation for a crew of four to six for $42-98$ days. The habitat serves as a base of operation for EVA activities and on-site robotic teleoperation. ${ }^{12}$ Like the LSAM, the habitat will include communications hardware to establish links to Earth, orbiting relays, and assets on the surface at short and long distances. The habitat design includes the following subsystems which coordinate through the surface network: airlock, habitat command and control, communications, life support, thermal control, power, crew accommodations, radiation protection, structures, and mechanisms. ${ }^{7,10}$

Exploration missions will employ a wide variety of science instruments to record data and analyze samples on-site. The science instrument package will include a seismometer, heat flow probe, laser retroflector, mass spectrometer, Lunar ejecta and meteorite detector, and interferometer. These instruments will possess the 
Table 1. Application protocols for the surface exploration network.

\begin{tabular}{|c|c|c|c|}
\hline Service & Type & Encoding/Compression & Transfer Protocol \\
\hline $\begin{array}{l}\text { Video (Real-time or } \\
\text { store-forward) }\end{array}$ & Constant Rate & $\begin{array}{l}\text { Raw, MPEG, MPEG-2, } \\
\text { MPEG-4 }\end{array}$ & $\begin{array}{l}\text { RTP, CRTP, CFDP, FTP, } \\
\text { HTTP, SMTP }\end{array}$ \\
\hline $\begin{array}{l}\text { Audio (Real-time or } \\
\text { store-forward }\end{array}$ & Constant Rate & $\begin{array}{l}\text { Raw, DPCM, ADPCM, } \\
\text { MPEG-3, Ogg, Flac }\end{array}$ & $\begin{array}{l}\text { RTP, CRTP, CFDP, FTP, } \\
\text { HTTP, SMTP }\end{array}$ \\
\hline File Transfer & Reliable & Zip, gzip, bzip & $\begin{array}{l}\text { CFDP, SMTP, FTP, HTTP, } \\
\text { NFS, SMB }\end{array}$ \\
\hline $\begin{array}{l}\text { Monitoring and } \mathrm{Au}- \\
\text { tomated Controls }\end{array}$ & Constant Rate, Control & & CBR (UDP) \\
\hline $\mathrm{TT} \& \mathrm{C}$ & Constant Rate & & CBR (UDP) \\
\hline $\begin{array}{l}\text { Robotic Command, } \\
\text { Control, and Collab- } \\
\text { oration }\end{array}$ & Control & $\begin{array}{l}\text { SSL, TLS, blowfish, des, } \\
\text { 3des, arcfou }\end{array}$ & CORBA, HTTP, XML \\
\hline $\begin{array}{l}\text { E-mail (text, audio, } \\
\text { video) }\end{array}$ & Reliable & ASCII, any file type & SMTP, POP, IMAP, CFDP \\
\hline
\end{tabular}

communications hardware to establish short distance $(<2 \mathrm{~km})$ surface-to-surface links and, should they be left on the surface by the crew, low rate direct to Earth links for science return after the mission has been completed.

Some tasks, such as construction, deployment of assets, and exploration of distant terrain, will be carried out by robots. ${ }^{7}$ These robots will range from teleoperated to autonomous and will contain a number of interconnected subsystems, including actuators, sensors, avionics, and power. They will possess the hardware to establish multiple simultaneous communications links to Earth, orbiting relays, and surface assets at short distances.

For exploration within a kilometer or two of a pressurized habitat, astronauts will use EVA suits. ${ }^{7}$ These suits will provide a number of remotely monitored, internally controlled subsystems, including life support (oxygen pressure, CO2 removal, thermal control, medical support), suit avionics (actuators, sensors, power, communications), and informatics (voice, video cameras, video displays, robotic system control). The EVA suit will provide the functionality for multiple simultaneous bidirectional communications paths and to assets on the surface at short range and to Earth.

The relatively short traverse range of humans in EVA suits can be augmented by the use of rovers to extend the range of exploration. ${ }^{7}$ The rover will transport the EVA crews over multi-kilometer distances and will also provide nearby exploration assets with network communications to surface assets at short and long distances, to orbiting relays, and to Earth.

The need will arise in long duration missions for human crews to explore beyond the range of an unpressurized rover. To support this capability a pressurized vehicle will be used which can provide simple crew habitation support for long duration excursions. ${ }^{7}$ It will contain the communications hardware required to establish multiple simultaneous bidirectional links to Earth, orbiting relays, and assets on the surface at short and long ranges. The pressurized vehicle will also contain a number of subsystems which can be remotely monitored and controlled, including personal communication modules, cameras, avionics, computer systems, life support, biological sensors, power systems, actuators and drivers, and portable workstations.

\section{Activities}

The tasks and activities which will be performed on the surface will be integral to the communications and networking architecture which is required. The exploration and science objectives to be performed on the surface can be broken into four categories: field work, telerobotic exploration, laboratory and intravehicular activity experiments, and preparation of materials for return to Earth. ${ }^{7}$

Key systems must be connected to the habitat upon landing, and routine inspections and repair will be performed on all critical systems. A substantial portion of the crew's time on the surface will be spent maintaining and possibly deploying systems to support exploration. This will require reliable and timely delivery of sensor data, and robotic commanding to deploy and pre-deploy assets. High fidelity images and 
Table 2. Surface exploration network technologies. Technologies used in the simulation are shown in bold italics. The technologies selected do not necessarily represent the best possible configuration.

\begin{tabular}{|c|c|c|c|}
\hline Service & Long Range ${ }^{*}$ Links & Medium Range ${ }^{\dagger}$ Links & Short Range ${ }^{ \pm}$Links \\
\hline Physical Layer & Ka-band, $S$-band & UHF, $S$-band & UHF, S-band, copper, fiber \\
\hline Data Link Layer & 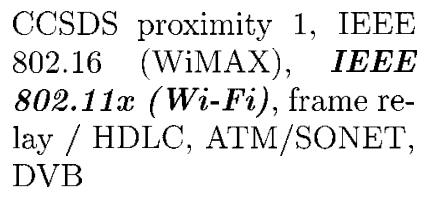 & $\begin{array}{l}\text { CCSDS proximity } 1, \boldsymbol{I} \boldsymbol{E} \boldsymbol{E} \boldsymbol{E} \\
\boldsymbol{8 0 2 . 1 1 x}(\boldsymbol{W} \boldsymbol{i}-\boldsymbol{F} \boldsymbol{i})\end{array}$ & $\begin{array}{l}\text { IEEE 802.3 (ethernet) } \\
\text { IEEE } 802.11 \times \text { (Wi-Fi), IEEF } \\
802.15 \text { (bluetooth), IEEF } \\
1394 \text { (Firewire), IEEE } 1355 \\
\text { (spacewire), FDDI, SOIF }\end{array}$ \\
\hline Network Layer & $\begin{array}{l}I P(\boldsymbol{R} F \boldsymbol{7 9 1}), \text { CCSDS } \\
\text { SCPS-NP, IPv6, AODV, } \\
\text { DSR, TORA, OSPF, RIP }\end{array}$ & $\begin{array}{l}\text { IP }(\boldsymbol{R F C} \mathbf{7 9 1}), \text { CCSDS } \\
\text { SCPS-NP, IPv6, AODV, } \\
\text { DSR, TORA, OSPF, RIP }\end{array}$ & $\begin{array}{l}\text { IP (RFC 791), CCSDS } \\
\text { SCPS-NP, spacecraft bus, } \\
\text { IPv6, } A O D V, \text { DSR, TORA, } \\
\text { OSPF, RIP }\end{array}$ \\
\hline $\begin{array}{l}\text { Transport Layer } \\
{ }^{*} 2-60 \mathrm{~km} \\
\dagger 0-2 \mathrm{~km} \\
\pm<10 \mathrm{~m}\end{array}$ & $U D P, \mathrm{TCP}, \mathrm{SCPS}-\mathrm{TP}$ & $U D P$, TCP, SCPS-TP & $U D P$, TCP, SCPS-TP \\
\hline
\end{tabular}

video could also be used to perform remote inspection and assessment. In addition, the presence of a crew on long duration missions will mandate health and medical operations as well as multimedia recreational and training programs to keep the crew in the best possible mental and physical health. ${ }^{10,13}$

Human presence will enable observations in exobiology, geology, and atmosphere to be made in the field. This field work will comprise geological observation and experimentation and sample curation. Samples and data will be collected and returned to the laboratory for analysis, and information will be transmitted to scientists on Earth so they can provide input. ${ }^{7}$ Scientists may also have real-time video conferencing with astronauts on the surface to coordinate experiments and sample curation. ${ }^{13}$

In order to explore the areas where it is dangerous or impossible for humans to travel, the crew will have the ability to operate telerobotic systems in real-time. These systems can be used to transport crew members to distant locales not easily accessible on foot or to perform unmanned robotic exploration over rough terrain. They will also be used in later missions to prepare and deploy surface infrastructure and equipment, including the power plant, in-situ resource utilization plant, and associated systems. This will require remote robotic command and control, as well as streaming video and sensor data to provide a rich virtual presence. . $^{73}$

On-site laboratory experiments will be an integral part of surface missions. Both geological and biological experiments will be carried out in a surface-based facility in collaboration with experts on Earth. ${ }^{13}$ Samples and data may be sent to scientists on Earth for real-time analysis and planning through video conferencing. ${ }^{7}$

\section{Applications}

The final crew task will be to select and package samples prior returning to Earth, where the samples will be studied in more detail. This may require experimental data to be sent to Earth prior to departure and video conferencing so that experts can provide advice on sample packaging. A large portion of this activity will involve collaboration with earth-based experts to select the samples which will be returned to Earth through video conferencing and exchange of experimental data. ${ }^{13}$

\section{Interfaces}

\section{Protocols and Networking}

The communications network nodes will interface with one another to enable cooperative and effective exploration activities. Applications will define the data which will be exchanged between communications endpoints, networking and protocols will ensure that the data is routed and delivered to its destination, and hardware and physical layer interfaces will create individual point-to-point wired and wireless links. 
Nodes on the surface will employ a diverse set of applications to enable highly coordinated and safe activities. ${ }^{14}$ These include real-time, bidirectional audio and video for conferencing, telemedicine, and recreation, high quality store-forward video for public outreach, data services for software uploads, e-mail, and downloads, and low jitter command for robotic teleoperation. Each major or critical exploration asset will use health monitoring and control systems to report its status in real-time, so that problems can be rapidly discovered and handled with minimal astronaut intervention.

The surface network will comprise a suite of protocols capable of reliable, efficient, on-demand, and bidirectional data delivery across multiple hops in the harsh Lunar environment. The protocols and network architecture used conform to the ISO/OSI model for layered communications and provide services for the transport, network, and data link layers.

In the transport layer, which regulates the end-to-end delivery of data, the User Datagram Protocol (UDP) protocol is used to provide simple, best effort data transport. Transport Control Protocol (TCP) was not selected due to its poor performance over high bandwidth, high delay networks. Applications which require zero packet loss use the CCSDS File Delivery Protocol ${ }^{15}$ application protocol over UDP.

On the network layer, which manages node addressing and data routing, the IP protocol is selected due to its widespread terrestrial use. The AODV mobile ad hoc networking (MANET) protocol was chosen to achieve dynamic ad hoc routing, although other protocols such as DSR or TORA could provide similar functionality. Routing functionality could also be provided by standard fixed routing protocols, although the effectiveness of that approach for surface networks has not been evaluated.

On the MAC/data link layer, which manages point-to-point communications including media access and framing, the protocols selected depend on the link type. Gigabit ethernet was used for all wired links, and a customized bent pipe relay protocol was used for space-based links. 802.11 variants were used for surface-to-surface links, including 802.11a (54 Mbps), 802.11b (11 Mbps), and 802.11g (54 Mbps).

\section{Hardware and Physical Layer}

Communications hardware on the surface will serve as data conduits, providing links upon which the protocols and applications can function. Because small and mobile nodes cannot accomodate communications hardware with large power, mass, and volume requirements, the hardware in the surface network will be as small, power-efficient, and light weight as possible. Communications hardware will consist of digital signal processors, transceivers, and antennas. SEU-tolerant digital signal processors will modulate/demodulate, encode/decode, and convert digital streams to analog RF (and vice versa). Power-efficient, wearable transceivers operating in the S-band will provide surface-to-surface transmit and receive capabilities, while Ka-band transceivers will operate over surface-to-orbit and in-space links. Small omnidirectional antennas are assumed for all surface-to-surface links due to their maturity, although developing technologies such as smart antennas may provide significant performance enhancement.

\section{Surface Network Simulation}

OPNET Modeler was used to simulate the surface network scenarios described above. The scenario, shown in figure 4, was implemented using a layered, hierarchical architecture. Because some of the basic capabilities for surface networks were not present in OPNET, tool modifications were performed to achieve simulation capability.

\section{A. Simulation Architecture}

The surface network was designed such that each surface-based entity (e.g. astronaut, habitat, rover, robot), was represented by a subnet. An EVA astronaut's subnet is called out in figure 4. Each host within that subnet represented an instrument, subsystem, or sensor. A wireless subnet was also created to contain the surface entity subnets and the links to orbiting relays and to Earth. This heirarchical architecture is inherently scalable and provides an easy means for the user to hide potentially distracting details.

\section{B. Tool Modifications}

Several modifications were made to enhance OPNET's capability to support surface communications networks for exploration. They include the development of the connectivity matrix, bent pipe relay support, 


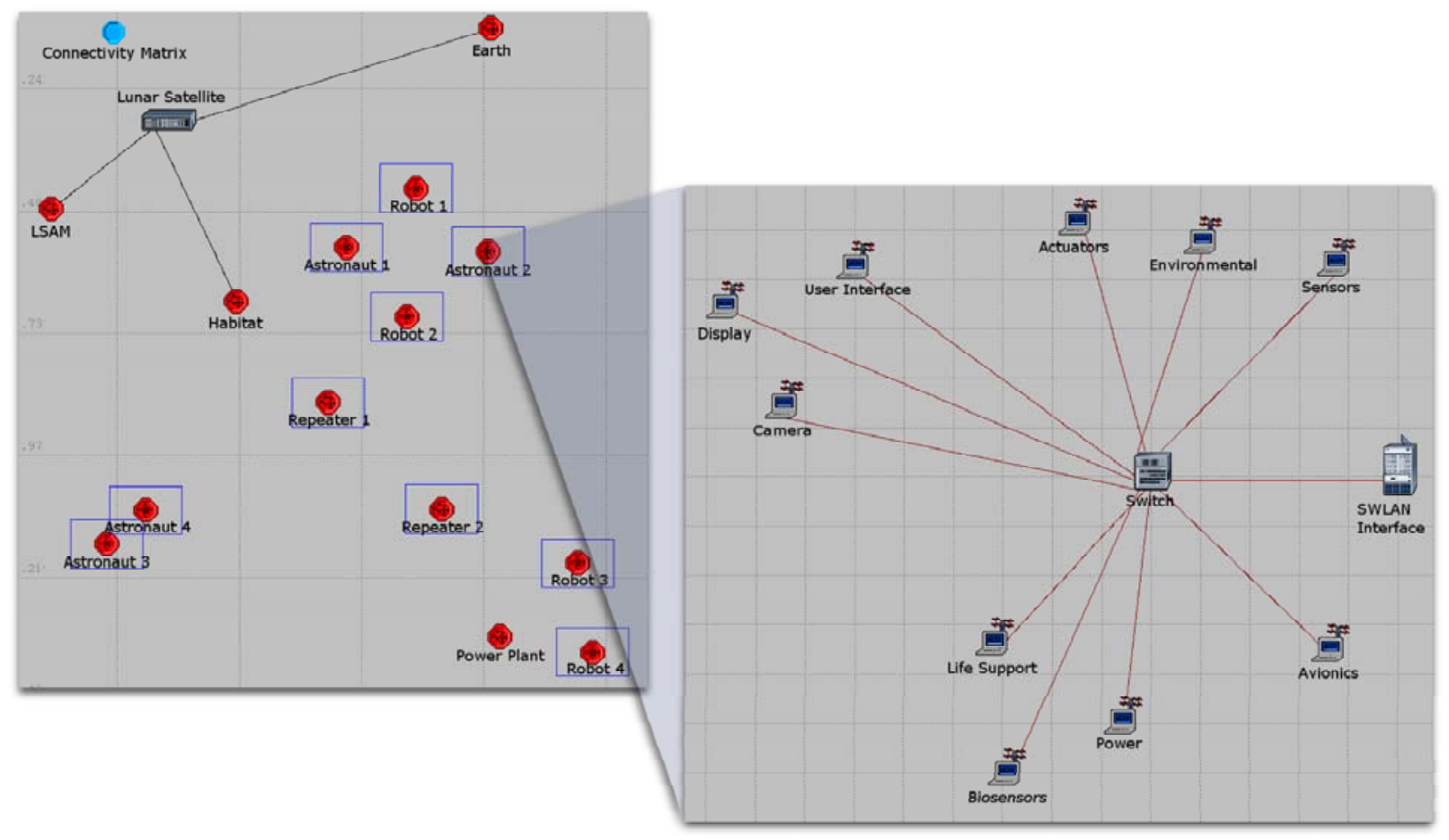

Figure 4. Lunar surface exploration scenario implemented in OPNET Modeler. The diagram on the left is the main SWLAN view and the diagram on the right is an EVA astronaut's personal area network.

CBR application, robotic control application, and reliable file transfer application.

The Space Communications Architecture called for three types of applications: constant bit rates (CBR), robotic control, and reliable file transfer. These applications could have been modeled using the standard OPNET Custom Application model, but the decision was put them in a new process model. There are two major reasons why this decision was made. First it allowed greater control over the presentation of results. Second, it allows delays to be added at the application layer. These delays will impact results without direct modeling of parts of the network (such as the backbone).

\section{Connectivity Matrix}

Over the course of routine field exploration, a number of unpredictable, unplanned events and effects induced by the surface terrain may occur resulting in signal degredation or loss. Multipath interference, for example, might drastically lower signal to noise ratio between two astronauts as they move relative to one another. A robot might explore into a cave or crater and completely lose line of sight (and its signal) with base camp. In both these cases, the network should be able to compensate for the weak or non-existant signal.

A connectivity matrix was developed to trigger these link changes from within a simulation. It enables the user to arbitrarily assign physical layer properties (line of sight, BER, delay, data rate) to links in surface exploration network scenarios. To enable this functionality, one process model and one node model were created, and four pipeline stages were modified. The connectivity matrix models can currently affect two types of links, satellite links and 802.11 radios. The models affect the two different links in very different ways, and the methods used to apply the connectivity matrix for these two links can be used as guide if there is a need to use other types of links in the future.

NoDE REQUIREMENTS The connectivity matrix models make use of the standard node attribute, "user id." It is expected that each "user id" value will initially be set to -1 . It is advised that every node model that is to be used have the "user id" hidden and set to -1 in the Node Interfaces dialog box (The Node Interface dialog box can be accessed under the Interfaces pull down menu in the node mode editor). At initialization the "user id" will be set on each node by the NASA_Connectivity_Setup process. After initialization pipeline 
stages will use the "user id" attribute to index the global connectivity matrix.

Global Connectivity Matrix The global connectivity matrix is a matrix containing connectivity information for each pair of nodes. It is assumed that there is only one connection between two nodes, and that links are unidirectional - a bidirectional link would be represented by two unidirectional links. The global connectivity matrix does not assume, expect, or require symmetry over links. This is intentional to allow for asymmetric links, particularly in regards to data rates. This also means that links could have asymmetric propagation delay even if this may not be physically possible. This allows for customization of link properties based on the hardware being modeled. The following information is contained in each connectivity information structure:

- Time - The time at which this set of information first becomes active.

- IDs - Transmitter and receiver IDs (also can be attained from array indexes).

- Connected - Boolean value indicating whether this is currently a valid link.

- Delay - Propagation delay between two nodes.

- BER - Bit Error Rate on the link between two nodes.

- Data Rate - The transmitter data rate for this link.

The third dimension of the array is used to hold the present and future connectivity information. The pipeline stages use the future and current connectivity information to linearly interpolate BER and propagation delay. If no future connectivity information is available the pipeline stages simply use the current BER and propagation delay. If connectivity information for a pair of nodes is never specified, default values calculated by Modeler's pipeline stages are used.

Setup Process Model The NASA_Connectivity_Setup process model sets up the global connectivity matrix for the simulation. There should be only one instance of this process model in a scenario, and it is most likely located in the in the NASA_Conn_Matrix node model. The global connectivity matrix is also declared in this process model. All pipeline stages that will use the connectivity matrix should extern it.

FUNCTIONALITY At the beginning of the simulation, this process reads in the connectivity input file specified in the connectivity matrix at-

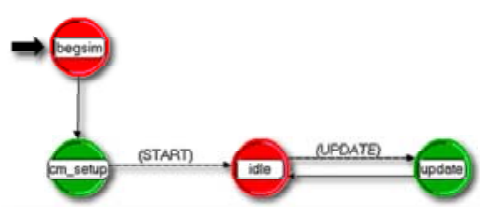

Figure 5. The NASA connectivity matrix setup process model. tributes. By parsing this file it creates the current connectivity matrix. A global event list is also created from the file for future updates for the connectivity matrix. This process model will use the event list to schedule self interrupts which trigger events to update the connectivity matrix.

For wired links this process model is responsible for changing the attribute "condition" on the link itself. This attribute will turn the link on and off, and its value will be controlled by the connectivity field of the connectivity information. All other values will simply be changed in the connectivity matrix.

\section{Bent-Pipe Relay}

During long duration, long range exploration, the need may arise to use orbiting relay satellites to extend the range of the surface network when surface-based line of sight becomes impractical. As a result, the characteristics of the relay satellites deployed can have a profound effect on surface network performance. A simple, "bent pipe" satellite system capable only of blindly forwarding packets over each of its communications interfaces, for example, could provide differing network and power efficiency than a satellite capable of intelligent switching among its interfaces at the packet level. 
To support modeling of bent pipe relay satellite systems, a simple MAC protocol was developed which blindly forwards any received data on all possible interfaces to simulate bent-pipe relays. The NASA Switch MAC model simply acts as both a dumb flooding switch and a simple MAC. In the end station's node model it will set below the IP ARP, and in the satellite switch's node model it connects all of the point-to-point transceivers. This process model is fairly simple. It has no attributes or output statistics.

If a packet comes to the NASA Switch MAC from a higher layer, the MAC encapsulates the packet with a MAC header. The header includes a unique packet identifier along with the source and destination IDs. Then the MAC sends the packet to every transceiver stream to which it is

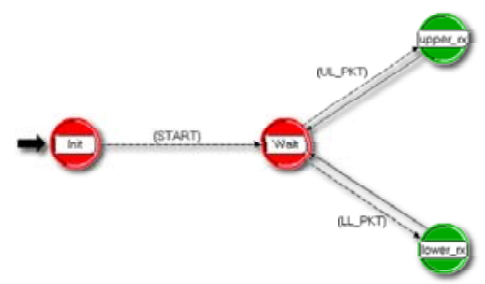

Figure 6. The simple MAC protocol model used for bent-pipe relays. connected.

When a packet comes to the NASA Switch MAC from any lower layer stream the following processing occurs. First the MAC checks the packet's unique ID and verifies that it has not received this packet before. If the MAC has previously received the packet then the packet is immediately destroyed. If the packet is new, the NASA Switch MAC will then check if this node is the final destination, and if so, it forwards the packet to higher layers. If the receiving node is not the final destination it will forward the packet on all interfaces except the one from which it received the original packet.

\section{Constant Bit Rate Application}

Many applications which will run over the surface network, such as streaming multimedia, tolerate moderate packet loss but depend on consistant latency and relatively constant data rates over time for proper functionality. To model these applications, a constant bit rate application (CBR) was developed to provide unreliable service with packets generated consistantly at a constant rate.

A custom model was built to simulate CBR traffic such as monitoring data and TT\&C. Video and voice are assumed to be CBR sources. The CBR application is the simplest of all the applications. The sending application sends a packet every $\mathrm{x}$ number of seconds so that the "Packet Size" and "Data Rate" attributes are true. All of the packet sizes are the

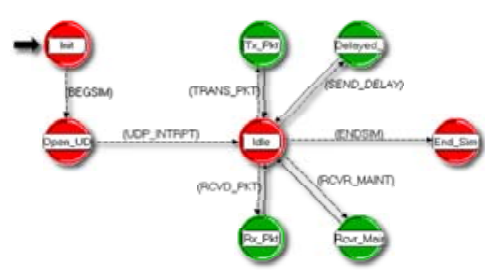

Figure 7. The NASA applications process model in OPNET modeler. constant "Packet Size" value specified in the object attributes, and the time between packet sends will also remain constant.

Packets are only sent between the application's start time and end time. The application's start time is randomly chosen using a uniform distribution to be between the times specified by the attributes, "Earliest Start Time" and "Latest Start Time." The application's end time is simply determined from the attribute "End Time." The receiving application simply receives the incoming packets and records the appropriate statistics.

\section{Reliable File Transfer}

The long distance and delays of space communication often cause problems from existing standard applications and protocols used over the internet. Standard OPNET file transfer applications such as FTP and HTTP assume TCP reliable transport, which is not feasible over Lunar distances. A simple model of CFDP, ${ }^{15}$ a file transfer protocol designed for high latency links was created to simulate reliable data transfer. CFDP is not modeled in OPNET's standard library, so a new model needed to be built.

CFDP is a file transfer protocol which assumes unreliable service from the transport layer. It was designed to handle the long delays associated with space communications. The CFDP model was designed to run over OPNET's standard UDP transport layer model. The deferred NACK was the CFDP flavor that was used. 


\section{Robotic Control}

A simple command/acknowledgement application was developed to model remote control of robotic mission elements. The Robotic Control application is also fairly simple. The basic concept is that the sender will send a robotic control message to the receiver. The receiver will receive the message and reply with an acknowledgement message. If the sender receives that acknowledgement then the sender will consider the message complete and schedule an interrupt for the next robotic control message. If the sender does not receive an acknowledgement then the sender will resend the robotic control message, and the cycle will repeat itself until either the sender receives an acknowledgement or sender the exhausts the maximum number of retries.

Robot control message sizes, the time between robotic commands, size of acknowledgements, and the maximum number of retries are determined by the user-defined attributes.

\section{Statistics}

Statistic collection for the above customized applications was also implemented. During a simulation, statistics were recorded both for every application stream and for every node. The node statistics represent the aggregate totals of the application streams running on the node.

\section{Application Statistics}

The application statistics recorded were:

- Offered Data Load - The amount of original data this application is trying to send. Control messages and resent data are not included in this statistic. The units are in bps.

- Offered Load - The load this application is offering to the lower layers. This statistic includes control messages, new data, and resent data. The units are in bps.

- Throughput - The amount of data received by the node. The units are in bps.

- Packets Sent - The total amount of packets sent by this node. The units are packets per second.

- Packets Received - The total amount of packets received by this node. The units are in packets per second.

- Delay - The amount of time it takes for a transmitted packet to reach its destination. The units are in seconds, and it is only recorded at the receiver.

\section{Node Statistics}

The node statistics recorded were:

- Total Offered Data Load - Sum of the application Offered Data Load.

- Total Offered Load - Sum of the application Offered Load.

- Total Throughput - Sum of the application Throughput.

- Total Packets Sent - Sum of the application Packets Sent.

- Total Packets Received - Sum of the application Packets Received.

- Delay - This compilation of the all the application delays.

\section{Simulation Results}


Table 3. Simulation statistics for different wireless protocols in a surface network environment.

\begin{tabular}{lll} 
Wireless Protocol & Arrival Probability & Jitter \\
\hline $802.11 \mathrm{~b}$ & 0.458 & 0.0502 \\
$802.11 \mathrm{~g}$ & 0.700 & 0.0438 \\
$802.11 \mathrm{a}$ & 0.778 & 0.0349
\end{tabular}

Simulations were carried out to assess the performance of the $802.11 \mathrm{a}$ and $802.11 \mathrm{~g}$ wireless protocols in the scenarios described above. The statistics were assessed based on average throughput (figure 8 ), end-to-end delay between surface-based nodes (figure 9), and total data dropped (figure 10 on the surface network.

In figure 8, 802.11g throughput drops slightly below that of $802.11 \mathrm{a}$, and $802.11 \mathrm{~b}$ produced the lowest average throughput, as expected due to its low maximum data rate of $11 \mathrm{Mbps}$. Both 802.11g and 802.11a scale their data rates according to the signal to noise ratio. $802.11 \mathrm{~g}$ has a smaller radius of 54 Mbps coverage than 802.11a, which accounts for the reduced throughput.

Table VI shows statistics collected during simulations for packet arrival probability (i.e. the probability that a packet will not be dropped) and jitter,

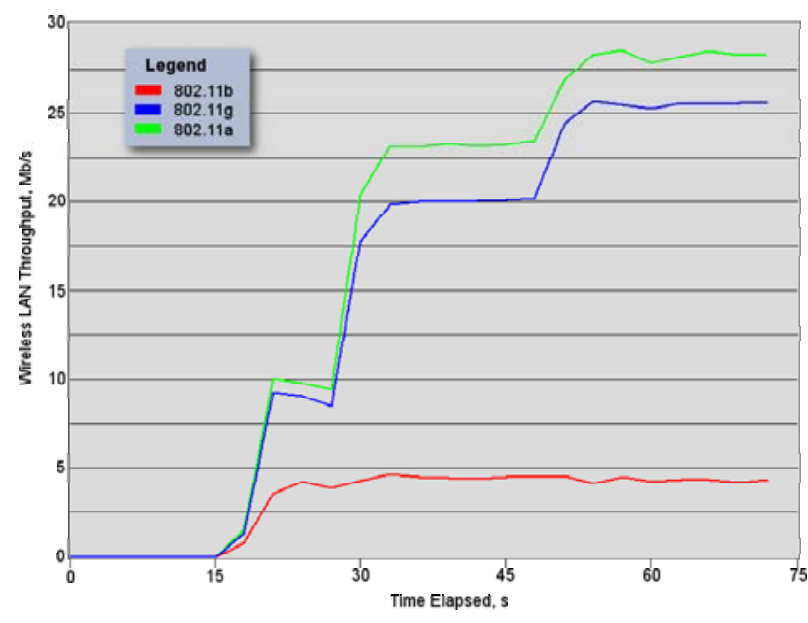

Figure 8. Average throughput on surface-to-surface communications links. which was calculated as the standard deviation of the end-to-end delay. 802.11a had the least packet drops, while $802.11 \mathrm{~b}$ had the most, due to the lower data rate. $802.11 \mathrm{~g}$ had more packet drops than 802.11 a due to the data rate scaling described above.

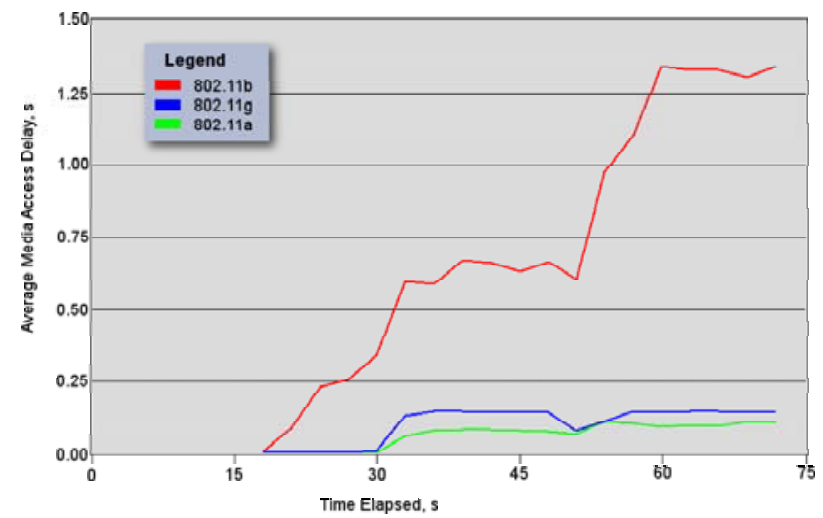

Figure 9. Surface network media access delay for nodes on the Lunar surface.

802.11a and 802.11g performed similarly with respect to media access delay. Because a saturated 802.11 network will generally experience higher media access delays, $802.11 \mathrm{~b}$ had the highest delays. For EarthMoon communications, the Earth-Moon backbone link dominates the end-to-end delay experienced by the users. While a more efficient routing protocol or media access protocol might be able to reduce delay on the surface, the improvements will only carry limited impact for improvement in total end-to-end delay. For surface-to-surface communications, however, delays of greater than one second can affect real-time use, and routing and media access improvements can greatly impact the user experience. 


\section{Conclusion}

This paper described modifications to the OPNET simulation tool to support surface communications networks for exploration. Simulation of surface networks provides a simple, inexpensive tool for architecture assessment, critical technology and capability gap analysis, and protocol evaluation which can play a crucial role at any stage in the capability and technology development cycles.

While this simulation tool is capable of modeling surface networks, there are several limitations to its fidelity. These limitations occur in three areas: physical layer modeling and RF propagation, protocols, and routing incompatibilities.

OPNET is limited at the physical layer for Lunar and Martian scenarios. In order to achieve realistic Lunar RF propagation effects, cross channel interference modeling, and implementations of modulation and coding schemes, OPNET must be augmented with an external modeling tool. Satellite

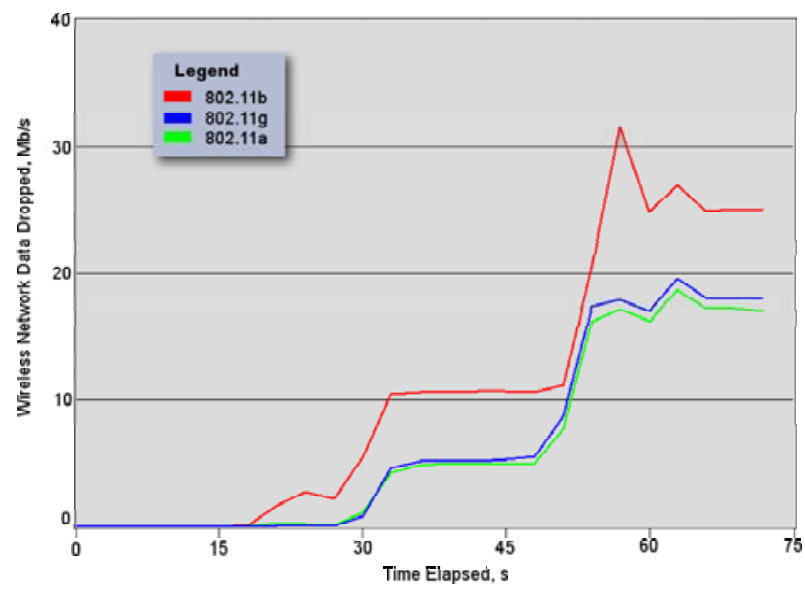

Figure 10. Data dropped by wireless network in simulated scenario. Tool Kit (STK) has been combined with OPNET for physical link modeling for satellite system simulation and terrestrial military excercise simulation, but the maturity of the interface is relatively low and may not scale well to include the Lunar surface. In addition, the OPNET 802.11 models break down at ranges greater than $300 \mathrm{~m}$. Surface network simulation is also limited by use of customized space protocols such as CCSDS and the SCPS protocol suite. Models for these protocols are not widely available. Finally, routing incompatibilities forced all interfaces in the simulation to run the AODV routing protocol, including fixed nodes on wired interfaces such as sensors and instruments, which may have led to sub-optimal performance. The next step in the development of high fidelity surface network simulation is to address these issues to create enhanced, realistic simulations to assist in the study, development, and evaluation process for surface systems.

\section{References}

${ }^{1}$ NASA, "The Vision for Space Exploration," http://www.nasa.gov/missions/solarsystem/explore_main.html, accessed september 27, 2004, Jan. 2004.

${ }^{2}$ NASA, "Exploration Crew Transportation System Requirements Document," NASA ESMD-RQ-0011, Feb. 2005.

${ }^{3}$ Soloff, J. A., Noreen, G., Deutsch, L., and Israel, D., "A Sustained Proximity Network for Multi-Mission Lunar Exploration," 1st Space Exploration Conference: Continuing the Voyage of Discovery, AIAA, Orlando, FL, Feb. 2005.

${ }^{4}$ Bhasin, K. B. and Hayden, J. L., "Developing Architectures and Technologies for an Evolvable NASA Space Communication Infrastructure," 22nd AIAA International Communications Satellite Systems Conference, AIAA, Monterey, CA, 2004.

${ }^{5}$ Bhasin, K. B. and Hayden, J. L., "Evolutionary Space Communications Architectures for Human/Robotic Exploration and Science Missions," Space Technology and Applications International Forum (STAIF), Institute for Space and Nuclear Power Studies, Albuquerque, NM, Feb. 2005.

${ }^{6}$ Schier, J. S., Rush, J. J., Williams, W. D., and Vrotsos, P., "Space Communication Architecture Supporting Exploration and Science: Plans and Studies for 2010-2030," 1st Space Exploration Conference: Continuing the Voyage of Discovery, AIAA, Orlando, FL, Feb. 2005.

${ }^{7}$ NASA, "Human Exploration of Mars: The Reference Mission of the NASA Mars Exploration Study Team," NASA special publication 6107 , July 1997.

${ }^{8}$ Bhasin, K. B., Linsky, T. W., Hayden, J. L., and Tseng, S., "Surface Communications Network Architectures for Exploration Missions," Space, AIAA, Long Beach, CA, Aug. 2005.

${ }^{9}$ NASA, "Robotic Lunar Exploration Program Requirements Document," NASA ESMD-RQ-0014, Feb. 2005.

${ }^{10}$ NASA, "Guidelines and Capabilities for Designing Human Missions," NASA/TM-2003-210785, Jan. 2003.

${ }^{11}$ Hanson, T. and Markley, R., "A Proposed Communications Architecture for the First Lunar Outpost," Globecom, IEEE, Houston, TX, 1993.

${ }^{12}$ Rapp, D. and Andringa, J., "Design Reference Missions for Human Exploration of Mars," International Space Development Conference, National Space Society, Washington, DC, May 2005.

${ }^{13}$ NASA, "The Mars Surface Reference Mission: A Description of Human and Robotic Surface Activities," NASA/TP2001-209371, Dec. 2001 
${ }^{14}$ Noreen, G. K., Cesarone, R. J., Deutch, L. J., and et al, "Integrated Network Architecture for Sustained Human and Robotic Exploration," Aerospace, IEEE, Big Sky, MT, March 2005.

${ }^{15}$ CCSDS, "CCSDS File Delivery Protocol (CFDP) Implementer's Guide," CCSDS 720.2-G-2, Sept. 2003. 